\title{
The Study of Stage Assessment Scheme of Programming Courses Based on Experiment
}

\author{
Dan-dan Zhao \\ School of Computer Science \& Engineering \\ Dalian Nationalities University \\ Dalian, China \\ zhaodd@yahoo.cn
}

\author{
Chun-li Xie \\ School of Electromechanical \& Information Engineering \\ Dalian Nationalities University \\ Dalian, China \\ chunlix@dlnu.edu.cn
}

\author{
Hai-yu Song \\ School of Computer Science \& Engineering \\ Dalian Nationalities University \\ Dalian, China \\ shy@dlnu.edu.cn
}

\begin{abstract}
Through analyzing the problems
in the assessment of computer programming courses, a stage assessment scheme based on experiments is presented in the paper. Experimental arrangement is consistent with theoretical progress, and experiment subjects are established according to theory course units in this scheme. At the same time, the result of the experiment is used to verify the study of the course. The proposed scheme improved the reliability and effectiveness of examination and assessment. Teaching effect is better than before and the professional quality of students is improved.
\end{abstract}

\section{Keywords-experiment; stage assessment; courses}

\section{INTRODUCTION}

Examination, assessment is an important part of higher education, aims at assessing their functional orientation; diagnostic and feedback function; education and excitation functions ${ }^{[1]}$. Our actual teaching, examination and assessment program is basically only completed its assessment of the students function as for the diagnosis and feedback, as well as education and incentives are difficult to reflect in a course. After half a semester of learning, the students finally get a good or bad score; this course will no longer be mentioned. Had not been revised to be rebuilt again remedy. This is very cruel for the children, now 90, is not conducive to their growth. Faced with this situation, should be explored as a precondition to develop students 'learning and application capabilities to develop students' capacity for sustainable development for the examination and assessment of the target program.

\section{PROBLEM IN COMPUTER PROGRAMMING COURSE EXAMINATION}

Although the syllabus is constantly revised, the teaching reform continue to explore, but the overall assessment of the computer programming courses basic or not student-centered, full attention to the cultivation of students' quality[2-4]. To sum up the problems are mainly the following:

\section{A. heavy theory and practice of light}

The computer is a very practical subject, programming courses regardless of the theories how clear, if you can not be converted them into code, all of this are pale. Our actual assessment often give students grades according to a theoretical exam papers accounting for $70 \%$ of the proportion of the semester end, this assessment way is not scientific.

\section{B. Single ability is tested by the examination and assessment}

Examination and assessment should point to the cultivation of students' ability to understanding, innovation, self-learning ability, practical ability and skills. But due to the long-term effects of cramming method of teaching ${ }^{[5]}$, more and more examination papers is to examine the students on basic concepts, memory and ability to understand basic principles, it is difficult to involve the application and improve.

\section{Grading credibility is not high}

Currently, the results of the computer programming courses are generally composed of two parts: the final exam and experimental results ${ }^{[6]}$. The above mentioned final exam that accounting for most of grade proportion, so we don't repeat them here. Experimental results generally accounted for approximately about $30 \%$ of the total score, which depends on the experiment testing, due to the constraints of time and resources, the checking process is basically to look at the program's final run effects, lack of experimental process control and code authenticity can not guarantee.

The new examination of the guiding ideology ${ }^{[1]}$ advocated: the examination to broaden the knowledge, improve the ability and quality as a whole, using a 
comprehensive, flexible, relative scoring method to detect the overall quality of students position to guide students diligent in thinking, creative thinking, smart development based on the understanding and mastery knowledge.

For the above, propose phase assessment program based on experimental in computer programming courses.

\section{STAGE ASSESSMENT PROGRAM BASED ON THE EXPERIMENTAL}

The experimental arrangement is consistent with theoretical progress, based on the experimental stage appraisal program to set up an experiment unit divided by theoretical lesson topic. Through the completion of the experiment to verify the theoretical grasp the situation. Quantify the three aspects of the following from the specific teaching preparation, experimental propulsion and assessment content specifically described.

\section{A. Teaching Preparation}

Teaching unit with experimental division corresponds to the theory and practice of teaching arrangements synchronization. The theory and practice of complementary, both in order to better promote each other. The teaching of computer science and technology does not allow too hasty, overnight. The student is not just access to a computer will be able to do the project. From the original $\mathrm{C}$ language variables, constants, data types to the sequence, branch, loop statement structure [7], from the software engineering requirements analysis, system design and realization of software testing [8], each teaching unit need to be coupled with corresponding examples, exercises plus the experimental job title, theoretical guidance to experiments, which in turn deepen the theoretical digestion experiments and understanding.

\section{B. Experimental propulsion}

Stages of assessment, the commit phase results. Preparation for each experiment or independent or interrelated, but each experiment by code, documentation, or the corresponding chart reporting stage results as the assessment is based on ${ }^{[9]}$. Software engineering, for example, the requirements phase can submit a requirements specification, design specification design phase commit, to achieve phase commit code, submit test reports the testing phase. The outcome should be in the form of multiple programming class code-based, engineering specifications, document-based domain knowledge allows students the stages of the learning experience through the form of briefing to share with you ${ }^{[10]}$. Experiment implementation of the order to give each student the results of each experiment.

\section{The assessment contents and quantify program}

Based on the experimental curriculum stage reaction of assessment can be a full range of content, such as: experimental attitude, ability, expression ability, innovation, information capture application capabilities. Reasonable distribution of various elements in the proportion of the entire examination is very important. The entire examination can be roughly divided into the usual performance, experimental process and final comprehensive three links. Usually can prepare from the attendance of courses and experiment, experiment of Investigation students learning attitude; experiment by the experimental results of each experiment, the demonstration effect, documentation and code normative assessment of the abilities of students, the expression ability and analysis problems and problem-solving skills; consider relatively comprehensive test at the end of student mastery of the whole course and integrated application level. Three links can enlarge the proportion of the experimental process, usually $5 \%-10 \%$, end of period accounted for $10 \%-20 \%$ the experimental process evaluation accounts for more than $70 \%$. Based on the experimental stage of the assessment of the overall program as shown in Table 1:

TABLE I. OVERALL SCHEME OF THE STAGE ASSESSMENT

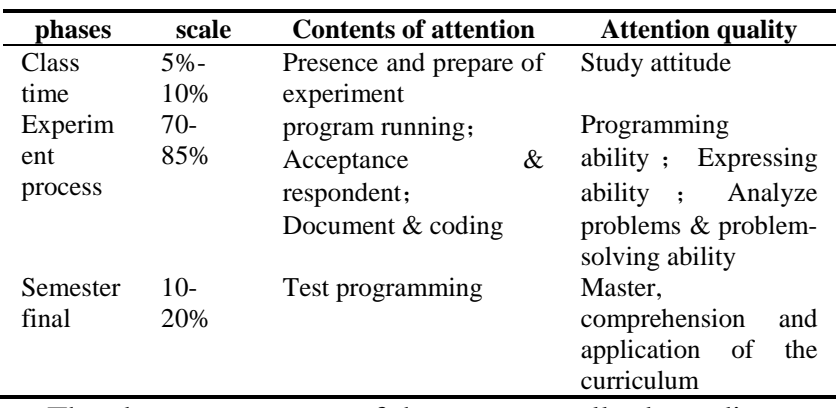

The above assessment of the stage, usually depending on student attendance, daily performance, answers to questions, classroom tests and other assessment scores. For the final grade, which should according to the teaching content and focus of the experimental arrangements in time to test the students' overall grasp of the courses. The results of the process evaluation, which will be $70 \%$ of the whole score, come from the cumulative accounting for the different proportion of multiple-stage experimental achievements. The following ASP.NET as the core content Web programming courses as an example to illustrate the results of the experiment quantization scheme. First, press the Table 2 divided experimental unit, and corresponding coefficient assigned.

TABLE II. THE DIVIDING OF ASP.NET EXPERIMENTAL UNIT

\begin{tabular}{clc}
\hline $\begin{array}{c}\text { Experiment } \\
\text { ID }\end{array}$ & \multicolumn{1}{c}{ Experiment name } & $\begin{array}{c}\text { coefficient } \\
(\boldsymbol{k})\end{array}$ \\
\hline 1 & Establishing publishing and configuring & 0.8 \\
2 & ASP.NET website & 0.8 \\
3 & The basis of develop ASP.NET website & 1 \\
4 & C\# \& ASP.NET 3.5 & 0.9 \\
5 & ASP.NET 3.5 Standard controls & 0.7 \\
6 & ASP.NET 3.5 Validation controls & 1 \\
7 & HTTP requests, responses, and state & 1.2 \\
8 & Danagement & 1.2 \\
9 & Data binding & 1.2 \\
\hline
\end{tabular}




\begin{tabular}{lll}
\hline & master pages \\
10 & Site navigation and site acceptance & 1.2 \\
\hline
\end{tabular}

Each of the above experimental results (Score) 10 points, the experimental cumulative multiplied by the total score is the experimental coefficient with the experimental achievements, that is $\sum_{i=1}^{n} S_{c o r e} \times k_{i}$. Wherein "Score" is each experiment Score, " $\mathrm{k}$ ” is the coefficient set according to the degree of importance of the experimental requirements, " $n$ " is the number of course experiment.

\section{CONCLUSIONS}

Based on the experimental program design course stage assessment scheme effectively plays the examination and diagnosis, feedback and education, incentive effect, and effectively improve the reliability and effectiveness of examination. Stage evaluation on students more fair and more advantageous, but it also gives teachers multiplies the workload of manual correction, all results will give teachers with great pressure. For this, we are developing and test operation auxiliary test system ${ }^{[14-15]}$.On the examination system under the support of teacher stress, can be greatly reduced, and the scheme of large-scale promotion foundation.

\section{REFERENCES}

[1] Xuejun Lin, WeiHua Xiong. Higher education examination system innovation and personnel training[J].Higher Science Education, 2007 (4) : 45-47.

[2] Xin Zhu. Historical review and value orientation of Educational philosophy of "Student-centered"[J]. Moden Education Management, 2012 (4) : 6 -9.

[3] LiFeng Zhao. Mathematical experiment course an important role in the quality of college students training[J].China University Teaching, 2011 (12) : 42-44.

[4] Ruifen Rong, Wenjie Yan, Jingxia Li, Chongxian Li. Exploration of assessment and evaluation mode for practice teaching courses[J]. Experimental Technology and Management,, 2011,28(3) : 232-234.

[5] Meiyan Hua. Research Application of PBL on Programming Courses[D].LiaoNing, Shenyang : Shenyang Normal University, 2012.

[6] Jing Chen, Li Niu, Liang Ye. 2011 Higher Vocational Education Electronic Information Professional Academic and Teaching Symposium[C]. Jiangsu,Wuxi:Scientific Research Publishing, USA, 2011-05-01:112-115.

[7] Ting Chen. Research on the experiment teaching reform of "C Language Programming" $[\mathrm{J}]$. Experimental Technology and Management, 2010, 27 (10) : 182-184.

[8] Yushen Cen , Fangmin Xiong. "Software Engineering" course project pedagogy research[J]. Computer Education,2009(11):83-85.

[9] Qing Hu, Jia Yu, Ling Chen, Yanchuan Wang. Research on Process Evaluation System of Specialized Courses of Automation[J]. Research and Exploration in Laboratory, 2010(10):296-299.

[10] Dandan Zhao, Xizuo Li, Haiyu Song, Lingfen Wang. Practice of project-driven teaching mode in computer personnel fostering $[\mathrm{J}]$. Experimental Technology and Management,2011, 28 (7) : 244247. 